Article

\title{
The effect of a pacer versus no-pacer on submaximal fitness test results among Special Olympics athletes
}

\author{
Viviene A. Temple ${ }^{*}$, Kendal F. Alston ${ }^{2}$, Jaymie J. Elder ${ }^{3}$, and Lynneth Stuart-Hill ${ }^{4}$
}

Received: 16 ${ }^{\text {th }}$ August 2018; Accepted: 11 $1^{\text {th }}$ March 2019; Published: $5^{\text {th }}$ April 2019

\begin{abstract}
Using a pacer when administering fitness tests reduces the practicality of testing. Additionally, presuming that a pacer is needed for all Special Olympics athletes is potentially discriminatory. We examined the need for a pacer to enhance performance and the test retestreliability of the six-minute walk test administered with a pacer (Criterion-m6MWT) and without a pacer (No-pacer 6MWT). Participants were $n=18$ Special Olympics athletes $($ men $=12$, Mean age $=37$ years $(S D=10.1)$ with low support needs. After familiarization, participants completed the Criterionm6MWT and the No-pacer 6MWT. The order of the tests was randomized. A week later, participants completed these tests again. There were no significant differences between any of the walk distances and both the Criterion-m6MWT and the No-pacer 6MWT had high test-retest reliability, intraclass correlation coefficients $=.90$ and .93 , respectively. The interclass correlation coefficients between the first administration of the Criterion-m6MWT and both of the No-Pacer tests were not as strong (i.e. $r=.65$ and $r=.65$ ) as the relationships between the second administration of the Criterion-m6MWT and both No-Pacer tests $(r=.81$ and $r=.87)$. These results suggest that adult Special Olympics athletes with relatively low support needs can perform the 6MWT without a pacer if the familiarization process is expanded to include a complete 6MWT.
\end{abstract}

Keywords: Intellectual disability, cardiorespiratory, psychometrics

\section{Introduction}

Higher levels of cardiorespiratory fitness are associated with lower rates of cardiovascular disease and all-cause mortality (Fogelholm, 2010; Zeno et al., 2010), fewer doctor and hospital visits (Mitchell, Gibbons, Devers, \& Earnest, 2004), and better athletic performance (Larsen, Nolan, Borch, \& Sondergaard, 2005). Among individuals with intellectual disability, cardiorespiratory fitness is predictive of mobility and daily functioning among older adults (Oppewal, Hilgenkamp, van Wijck, Schoufour, \& Evenhuis, 2014) and levels of body fat among adolescents (Salaun \& Berthouze-Aranda, 2012).

In the field, such as during sports practices and monitoring fitness program results, cardiorespiratory fitness is often assessed using a submaximal test (American College of Sports Medicine, 2017). This type of test is typically administered because measuring cardiorespiratory fitness directly (i.e. using respired gas analysis while a person exercises) is often not feasible or practical. Direct measurement of cardiorespiratory fitness requires expensive equipment, welltrained personnel, considerable time, as well as a participant capable of, and willing to, give maximal effort. Submaximal test protocols using a wide variety of exercise modes have been developed, however, a walking test may be preferred for individuals with intellectual disability as walking is common form of physical activity (Dairo, Collett, Dawes, \& Oskrochi, 2016; Draheim, Williams, \& McCubbin, 2002; Temple \& Walkley, 2003).

The six-minute walk test (6MWT, American Thoracic Society, 2002) is a submaximal test that involves walking as quickly as possible on a flat, hard surface, for a period of six minutes. Standard phrases of encouragement are provided at the end of each minute and there is no warm-up. The 6MWT has been widely used as a test of submaximal exercise test among individuals living with many different conditions, including: Alzheimer disease (Ries, Echternach, Nof, \& Gagnon Blodgett, 
2009), osteoarthritis (Bingchen et al., 2008), severe heart and lung disorders (Brown \& Wise, 2007), Parkinson's disease (Falvo \& Earhart, 2009), cerebral palsy (Fitzgerald, Hickey, Delahunt, Walsh, \& O'Brien, 2016), and cystic fibrosis (Gruet, Brisswalter, Mely, \& Vallier, 2010).

The concurrent validity of the 6MWT with the graded maximal treadmill test has been examined among adults (Guerra-Balic et al., 2015; Nasuti, Stuart-Hill, \& Temple, 2013) and older adults (GuerraBalic et al., 2015) with intellectual disabilities. Because task understanding and motivation can affect fitness test results among adults with intellectual disabilities (Kittredge, Rimmer, \& Looney, 1994; Lavay, Reid, \& Cressler-Chaviz, 1990), Nasuti et al. incorporated several accommodations to enhance comprehension, motivation, and maximal effort without changing the performance requirements of the test. Consistent with previously reported fitness testing protocols for adults with intellectual disability (e.g. Pitetti \& Fernhall, 2005; Rintala, McCubbin, \& Dunn, 1995), a pacer was provided and participants were familiarized with the protocol, equipment, environment, and staff. The pacer walked $1-3$ metres ahead of the participant and standardized phrases of encouragement were provided every 15-seconds. This version of the test, referred to as the modified six-minute walk test (m6MWT), has excellent feasibility and test-retest reliability and substantial relationships with peak oxygen uptake (VO2peak) among adults with intellectual disabilities (Nasuti et al., 2013). The strong test-retest reliability and feasibility of the 6MWT when a pacer is used has also been demonstrated among adolescents with intellectual disabilities (Casey, Wang, \& Osterling, 2012) and those with severe intellectual and sensory disabilities (Waninge, Evenhuis, van Wijck, \& van der Schans, 2011).

Using a pacer when administering the m6MWT increases the number of personnel needed to conduct the test and possibly the costs and practicality of testing. In addition, presuming that a pacer is needed for all individuals with an intellectual disability to perform the test is potentially discriminatory (Iacono, 2006). To increase the utility of the 6MWT, we compared participants' performance on the 6MWT with a pacer (Criterion-m6MWT) and without a pacer (No-Pacer 6MWT). The two versions of the test were defined as:

- Criterion-m6MWT: participants walk as far as possible in six minutes. Walking back and forth around the orange cones set 30-metres apart. A research assistant walks a little bit in front of the participant (1:1 pacer) and provided encouragement every 15 seconds.

- $\quad$ No-Pacer 6MWT: as per the Criterion-m6MWT, but without the use of a pacer.

With a population of adult Special Olympics athletes, the specific research questions addressed in this study were 1) What is the relationship between the No-Pacer 6MWT with the Criterionm6MWT? 2) What is the test-retest reliability of the No-Pacer 6MWT?

\section{Materials and Methods}

\section{Participants}

Volunteer participants were recruited through Special Olympics in Victoria, British Columbia, Canada. Special Olympics is a global organization that provides opportunities for sport, competition, health screenings, and health promotion activities to more than five million athletes with intellectual disabilities in 172 countries (Special Olympics, 2016). A person is eligible to participate in Special Olympics if he/she has an intellectual disability as determined by meeting any of the following requirements 1) they have a cognitive delay as determined by standardized measures such as an intelligence quotient (IQ), 2) an agency or professional has determined the person has an intellectual disability in accordance with local policies, or 3) the person has functional limitations in both general learning (such as IQ) and in adaptive skills (Special Olympics Inc., 2012).

The exclusion criteria for this study were 1) individuals were not ambulatory, 2) they used an assistive mobility device, or 3) subsequent to Physical Activity Readiness Questionnaire (PAR-Q) (Canadian Society for Exercise Physiology, 2002) screening indicating a need for follow-up, the individual did not receive medical clearance to participate.

Approval for this study was granted by the Human Research Ethics Board of the University of Victoria BC, Canada, and by SOBC, protocol number 13-518. Potential participants were shown a short video-clip depicting what was involved in the study at a Special Olympics practice. If the 
individual was interested in participating, written informed consent was obtained from each athlete or the participant's legal guardian. When consent was obtained from a guardian, the athlete provided assent.

\section{Measures}

\section{The Six-Minute Walk Tests}

The modified six-minute walk test (m6MWT) (Nasuti et al., 2013) was used as the Criterion for this study (i.e. Criterion-m6MWT). Nasuti et al. demonstrated that the m6MWT has adequate concurrent validity $\left(R^{2}=0.67\right)$ with The Graded Maximal Treadmill Test (Fernhall \& Tymeson, 1988) and strong test-retest reliability (ICC $=0.98$ ) among adults with intellectual disability. The test involved walking as quickly as possible for six minutes on an indoor flat and hard surface, along a straight 30-metre path, and around a cone at each end of the path. A pacer (ratio of 1 participant to 1 pacer) walked $1-3$ meters ahead of the participant and the pacer provided standard phrases of encouragement e.g. "you're doing well" every 15-seconds. At the end of each minute, the pacer indicated how many minutes to go. The path was marked at 2-metre intervals with floor tape, and at the completion of the test, the research assistant timing and recording laps, placed a piece of tape at the heel of the last step taken by the participant. In this way, total distance walked in meters was counted. The m6MWT also had a brief familiarization phase consisting of 1) a demonstration of two laps ( $30 \mathrm{~m} \times 2)$ of the test by the pacer, and 2) a practice of two laps by the participant with the pacer (30m $\times 2)$. The only changes to the m6MWT for the "No-pacer" condition was the removal of the pacer. The additional verbal encouragement (compared to the original American Thoracic Society (2002) version of the test) and familiarization remained.

\section{The Physical Activity Readiness Questionnaire (PAR-Q)}

The PAR-Q (Canadian Society for Exercise Physiology, 2002), completed by the participant if they provided consent or a caregiver if they provided assent, was used to prescreen potential participants to determine if further clearance was needed from a physician before the participant was enrolled in the study.

\section{Supports Intensity Scale (SIS)}

The SIS (Thompson et al., 2004) measures support needs in six activity categories: home living, community living, lifelong learning, employment, health and safety, and socializing. The assessment assists parents/guardians, service providers, healthcare professionals, and coaches and the like, tailor supports for individuals with intellectual disabilities. Construct-, content-, and criterion-related validity have been established with intelligence and adaptive behaviour (Thompson et al., 2004). The SIS standard score is the sum of the standard scores from all of the subscales, and represents a general indication of the amount and intensity of the support needed. The higher the reported standard scores and percentiles, the greater the amount of support needed. Participants provided the responses to the SIS alongside a person who knew them well. As per the SIS administration protocol, the SIS was administered by one of the research team with the assistance of parents, care providers, coaches, or employers who knew a participant well.

\section{Participant information}

Additional information, specifically: age and date of birth, years of participation with Special Olympics, and contact information, was obtained from the participants or a parent/guardian. With shoes and excess clothing (e.g. coats and jackets), removed, participant height was then measured using a portable stadiometer to the nearest $0.1 \mathrm{~cm}$ and body mass was measured on a digital scale to the nearest $0.1 \mathrm{~kg}$. 


\section{Procedures}

A descriptive correlational design was used to establish the relationship between the Criterionm6MWT and the No-Pacer 6MWT. The test-retest reliability of the Criterion-m6MWT and the NoPacer 6MWT were also determined. All testing occurred at the University of Victoria. A long and straight hallway was used for the walking tests and a laboratory space was utilized to measure height and weight and to administer the SIS.

\section{Familiarization}

During participants' first visit to the University, consent and assent (if applicable) forms were collected, the SIS was administered, and weight and height were measured. Subsequently, participants were then shown the Criterion-m6MWT by a member of the research team, and then they practiced several laps of the test with and without a pacer.

\section{Walking Tests}

One week after the familiarization visit, participants completed the Criterion-m6MWT and the No-pacer 6MWT, separated by a 30-minute rest period with juice and/or water provided. To control for the effect of testing the order of the tests was randomized. The walking tests were repeated a week later in the reverse order.

\section{Data analyses}

Descriptive statistics (mean, standard deviation, minimum, and maximum) were computed for age, SIS, weight, height, BMI, and distances walked. Reliability analysis (Field, 2013) was used to compute intraclass correlation coefficients (ICC, Cronbach, 1951; Field, 2013) to establish the test retest reliability of the Criterion-m6MWT and the No pacer-6MWT. Interclass correlation coefficients (Pearson's $r$ ) were used to compute the relationship between the Criterion-m6MWTs and the Nopacer 6MWTs. Analyses of variance (ANOVA) were used to examine differences in the distance walked for each comparison (i.e. Criterion1-m6MWT with No-pacer1 6MWT, Criterion1-m6MWT with No-pacer2 6MWT, Criterion2-m6MWT with No-pacer1 6MWT, and Criterion2-m6MWT with No pacer2 6MWT). All analyses were performed using IBM SPSS Statistics Version 24.

\section{Results}

The aim of this study was to examine the need for a pacer to enhance performance and the test retest-reliability of the m6MWT administered with and without a pacer. As can be seen in Table 1, the participants were $n=18$ adult Special Olympics athletes (men, $n=12$ ) with a mean age of 37 years. After screening with the PAR-Q, three participants were referred to their doctor; all received medical clearance to participate. Individual support needs as measured by the Supports Intensity Scale (SIS; Thompson et al., 2004) were low, ranging from $<1 \%$ to $14 \%$.

Table 2 provides the Pearson's correlation coefficients for the relationships between each of the Criterion-m6MWTs and each of the No-pacer 6MWTs. All of the correlation coefficients were significant, ranging from $r=.65$ to $r=.87$. There were no significant differences between the comparison pairs as evidenced by the ANOVA results, specifically: Criterion1-m6MWT with Nopacer1 6MWT $(p=.54)$, Criterion1-m6MWT with No-pacer2 6MWT $(p=.44)$, Criterion2-m6MWT with No-pacer1 6MWT $(p=.23)$, and Criterion2-m6MWT with No-pacer2 6MWT $(p=.08)$. Both the Criterion-m6MWT and the No-pacer 6MWT had high test-retest reliability, ICC $=.90$ and .93 , respectively. 
Table 1. Mean and range of scores for participant $(n=18)$ characteristics and 6MWT distances

\begin{tabular}{lcccc}
\hline Variable & Minimum & Maximum & Mean & \multicolumn{1}{c}{ SD } \\
\hline Age (years) & 19 & 58 & 36.6 & 10.1 \\
SIS Score (\%) & $<1$ & 14 & 5.1 & 4.6 \\
SIS Support Needs Index* & 56 & 84 & 66.1 & 8.9 \\
Weight (kg) & 49.9 & 133.8 & 81.0 & 20.4 \\
Height (cm) & 153.9 & 185.4 & 169.6 & 2.4 \\
BMI (kg/m $\left.{ }^{2}\right)$ & 19.0 & 39.5 & 28.1 & 5.8 \\
6MWT distances & & & & \\
$\quad$ Criterion test \#1 (m) & 465.6 & 747.0 & 601.0 & 80.6 \\
$\quad$ Criterion test \#2 (m) & 477.3 & 737.8 & 605.9 & 72.9 \\
$\quad$ No Pacer test \#1 (m) & 457.2 & 745.2 & 592.4 & 75.8 \\
$\quad$ No Pacer test \#2 (m) & 486.0 & 767.6 & 588.6 & 77.6 \\
\hline
\end{tabular}

Note. ${ }^{*}$ Data collected on $n=16$ participants.

Table 2. Relationships (Pearson's r) between m6MWT distances for tests administered with and without a pacer on two occasions for each test

\begin{tabular}{lcc}
\hline & No_Pacer_01 & No_Pacer_02 \\
\cline { 2 - 3 } Criterion_01 & $.65^{* *}$ & $.65^{* *}$ \\
Criterion_02 & $.81^{* *}$ & $.87^{* *}$ \\
\hline
\end{tabular}

Note. Correlation is significant at the 0.01 level (2-tailed).

\section{Discussion}

Participants in this study performed the m6MWT and the No-pacer 6MWT twice, and both versions of the test had excellent test-retest reliability. These results suggest that either version of the test could be used to monitor change in the cardiorespiratory fitness level of Special Olympics athletes over time. There were no significant differences when the Criterion-m6MWT (with a pacer) distances walked were compared to distances walked without a pacer. Additionally, the relationships between the Criterion2-m6MWT and both of the No-Pacer tests were strong. However, the relationships between the Criterion1-m6MWT and both of the No-Pacer tests (i.e. $r=.65$ and $r=.65$ ) were not as strong as the relationships between the second administration of the Criterion-m6MWT and the NoPacer tests (i.e. $r=.81$ and $r=.87$ ). The difference in the strength of the correlation coefficients between the first and second administration of the Criterion-6MWT and the No-pacer versions of the tests, may in part be explained by the greater variability in distances walked the first time participants performed a complete Criterion-6MWT. This finding suggests that the familiarization phase of using either the m6MWT or a No-pacer version of the test with adults with intellectual disabilities should include a complete 6MWT. This complete 6MWT would be in addition to the familiarization steps of observing someone doing the test and completing a several 30-metre laps of the test to be performed (i.e. with or without a pacer). This result is consistent with the findings of Casey et al. (2012) who was investigating the test-retest reliability of the 6MWT with a pacer following children and young adults with Down syndrome. When these researchers administered the 6MWT four times over a twoweek period, they found the ICC for the four tests was $r=.84$, however when the first test was omitted the ICC increased to $r=.94$.

There are certain limitations to the present study. Our sample was small, and participants in this study were healthy, motivated, relatively young adult Special Olympics athletes with low support needs, which limits generalizability. It is possible that older adults and those with higher support needs will not respond in the same way to the removal of the pacer. The findings of this study should be replicated with individuals with greater needs for support. 


\section{Conclusions}

Cardiorespiratory fitness is an important health outcome measure and a sensitive measure of changes in response to physical activity (American College of Sports Medicine, 2017), and the 6MWT has been widely used to assess fitness in community and clinical groups (e.g. Møller et al., 2018; Serra et al., 2015). In addition, versions of the 6MWT have been shown to be feasible among individuals with intellectual disabilities (Boer \& Moss, 2016; Guerra-Balic et al., 2015; Waninge et al., 2011). The results of this study suggest that with familiarization, that includes a complete 6MWT and encouragement every 15-seconds, adult Special Olympics athletes with relatively low support needs can complete the $6 \mathrm{MWT}$ without a pacer. With or without a pacer, the 6MWT is straightforward, with few time, space, measurement, and equipment requirements. However, the No pacer 6MWT has fewer demands for personnel to administer the test than the m6MWT.

\section{Perspectives}

Using a pacer when administering fitness tests reduces the practicality of testing, and presuming that a pacer is needed for all Special Olympics athletes is potentially discriminatory. We found that adult Special Olympics athletes with relatively low support needs could perform the six-minute walk test (6MWT) without a pacer with a high degree of consistency. Further, the results were strongest after a familiarization process and one complete performance of the 6MWT.

\section{Author Affiliations:}

1 School of Exercise Science, Physical and Health Education, University of Victoria, British Columbia, Canada

2 Community Supports, BC Ministry of Health; Kendal.Alston@gov.bc.ca

3 School of Exercise Science, Physical and Health Education, University of Victoria, British Columbia,

Canada; jelder@uvic.ca

4 School of Exercise Science, Physical and Health Education, University of Victoria, British Columbia,

Canada; Istuhilll@uvic.ca

*Correspondence: vtemple@uvic.ca; Tel.: +01-250-721-7846

Author Contributions: Conceptualization, VT, KA and LSH.; Methodology, VT, KA, JE, LSH; Formal Analysis, VT, JE; Investigation, VT, KA, JE, LSH; Resources, VT, LSH Data Curation, VT, KA, JE,; Writing-Original Draft Preparation, VT.; Writing-Review \& Editing, VT, KA, JE, LSH; Supervision, VT, LSH.; Project Administration, VT, KA, JE.

Funding: This research received no external funding

Acknowledgments: We wish to thank Special Olympics Victoria, BC for their assistance with recruitment of participants.

Conflicts of Interest: The authors declare no conflict of interest.

\section{References}

American College of Sports Medicine. (2017). ACSM's Guidelines for Exercise Testing and Prescription (Vol. 10). Baltimore, MD: Lippincott, Williams \& Wilkins.

American Thoracic Society. (2002). ATS statement: Guidelines for the six-minute walk test. American Journal Of Respiratory And Critical Care Medicine, 166, 111-117.

Bingchen, A., Kerong, D., Zhenan, Z., You, W., Yongqiang, H., Tingting, T., \& Huanqing, Y. (2008). Baduanjin alleviates the symptoms of knee osteoarthritis. Journal of Alternative E Complementary Medicine, 14, 167-174.

Boer, P. H., \& Moss, S. J. (2016). Validity of the 16-metre PACER and six-minute walk test in adults with Down syndrome. Disability and Rehabilitation, 38(26), 2575-2583. doi:10.3109/09638288.2015.1137982

Brown, C. D., \& Wise, R. A. (2007). Field tests of exercise in COPD: The six-minute walk test and the shuttle walk test. Journal of Chronic Obstructive Pulmonary Disease, 4, 217-223.

Canadian Society for Exercise Physiology. (2002). Physical Activity Readiness Questionnaire - PAR-Q. In. Retrieved from http://www.csep.ca/CMFiles/publications/parq/par-q.pdf

Casey, A. F., Wang, X., \& Osterling, K. (2012). Test-retest reliability of the 6-Minute Walk Test in individuals with Down Syndrome. Archives of Physical Medicine E Rehabilitation, 93(11), 2068-2074. 
Cronbach, L. (1951). Coefficient alpha and the internal structure of tests. Psychometrika, 16, 297-334.

Dairo, Y. M., Collett, J., Dawes, H., \& Oskrochi, G. R. (2016). Physical activity levels in adults with intellectual disabilities: A systematic review. Preventive Medicine Reports, 4, 209-219. doi:10.1016/j.pmedr.2016.06.008

Draheim, C. C., Williams, D. P., \& McCubbin, J. A. (2002). Prevalence of physical inactivity and recommended physical activity in community-based adults with mental retardation. Mental Retardation, 40, 436-444.

Falvo, M. J., \& Earhart, G. M. (2009). Six-minute walk distance in person with Parkinson's disease: A hierarchical regression model. Archives of Physical Medicine and Rehabilitation, 90, 1004-1008.

Fernhall, B., \& Tymeson, G. T. (1988). Validation of cardiovascular fitness field tests for adults with mental retardation. Adapted Physical Activity Quarterly, 5(1), 49-59.

Field, A. (2013). Discovering Statistics Using IBM SPSS Statistics (4 ed.). Thousand Oaks,CA: SAGE.

Fitzgerald, D., Hickey, C., Delahunt, E., Walsh, M., \& O'Brien, T. (2016). Six-minute walk test in children with spastic cerebral palsy and children developing typically. Pediatric Physical Therapy, 28(2), 192-199. doi:10.1097/pep.0000000000000224

Fogelholm, M. (2010). Physical activity, fitness and fatness: relations to mortality, morbidity and disease risk factors. A systematic review. Obesity Reviews, 11, 202-221.

Gruet, M., Brisswalter, J., Mely, L., \& Vallier, J. M. (2010). Use of the peak heart rate reached during six-minute walk test to predict individualized training intensity in patients with cystic fibrosis: Validity and reliability. Archives of Physical Medicine and Rehabilitation, 91, 602-607.

Guerra-Balic, M., Oviedo, G. R., Javierre, C., Fortuno, J., Barnet-Lopez, S., Nino, O., . . . Fernhall, B. (2015). Reliability and validity of the 6-min walk test in adults and seniors with intellectual disabilities. Research in Developmental Disabilities, 47, 144-153. doi:10.1016/j.ridd.2015.09.011

Iacono, T. (2006). Ethical challenges and complexities of including people with intellectual disability as participants in research. Journal of Intellectual $\mathcal{E}$ Developmental Disability, 31(3), 173-179. doi:10.1080/13668250600876392

Kittredge, J. M., Rimmer, J. H., \& Looney, M. A. (1994). Validation of the Rockport Fitness Walking Test for adults with mental retardation. Medicine and Science in Sports and Exercise, 26, 95-102.

Larsen, H. B., Nolan, T., Borch, C., \& Sondergaard, H. (2005). Training response of adolescent Kenyan town and village boys to endurance running. Scandinavian Journal of Medicine and Science in Sports and Exercise, 15, 4857.

Lavay, B., Reid, G., \& Cressler-Chaviz, M. (1990). Measuring the cardiovascular endurance of persons with mental retardation: a critical review. Exercise \& Sport Sciences Reviews, 18, 263-290.

Mitchell, T. L., Gibbons, L. W., Devers, S. M., \& Earnest, C. P. (2004). Effects of cardiorespiratory fitness on healthcare utilization. Medicine and Science in Sports and Exercise, 36(12), 2088-2092.

Møller, T. K., Nielsen, T. T., Andersen, R., Lundager, I., Hansen, H. F., Ottesen, L., . . Randers, M. B. (2018). Health effects of 12 Weeks of team-sport training and fitness training in a community health centre for sedentary men with lifestyle diseases. BioMed Research International, 1-9. doi:10.1155/2018/1571807

Nasuti, G., Stuart-Hill, L., \& Temple, V. A. (2013). The six-minute walk test for adults with intellectual disability: A study of validity and reliability. Journal of Intellectual Disability Research, 38(1), 31-38.

Oppewal, A., Hilgenkamp, T. I. M., van Wijck, R., Schoufour, J. D., \& Evenhuis, H. M. (2014). Physical fitness is predictive for a decline in daily functioning in older adults with intellectual disabilities: Results of the HAID study. Research in Developmental Disabilities, 35(10), 2299-2315. doi:10.1016/j.ridd.2014.05.027

Pitetti, K. H., \& Fernhall, B. (2005). Mental retardation. In J. S. Skinner (Ed.), Exercise testing and exercise prescription for special cases: Theoretical basis and clinical application (3rd ed.). Philadelphia: Lippincott Williams \& Wilkins.

Ries, J. D., Echternach, J. L., Nof, L., \& Gagnon Blodgett, M. (2009). Test-retest reliability and minimal detectable change scores for the timed "up \& go" test, the six-minute walk test, and gait speed in people with Alzheimer disease. Physical Therapy, 89, 569-579.

Rintala, P., McCubbin, J. A., \& Dunn, J. M. (1995). Familiarization process in cardiorespiratory fitness testing for persons with mental retardation. Sports Medicine, Training, and Rehabilitation, 5, 15-27.

Salaun, L., \& Berthouze-Aranda, S. E. (2012). Physical fitness and fatness in adolescents with intellectual disabilities. Journal of Applied Research in Intellectual Disabilities, 25(3), 231-239. doi:10.1111/j.14683148.2012.00659.x

Serra, A. J., de Carvalho, P., Lanza, F., de Amorim Flandes, C., Silva, S. C., Suzuki, F. S., . . Silva, J. A. (2015). Correlation of six-minute walking performance with quality of life is domain- and gender-specific in healthy older adults. PLOS ONE, 10(2), 1-8. doi:10.1371/journal.pone.0117359

Special Olympics. (2016). Special Olympics Annual Report. In (pp. 23). Retrieved from http://www.specialolympics.org/Common/Reach Report.aspx 
Special Olympics Inc. (2012). Special Olympics Official General Rules. In (pp. 126). Retrieved from http://media.specialolympics.org/soi/files/resources/GeneralInformation/General\%20Rules/AmendedGeneralRules02_FINAL.pdf

Temple, V. A., \& Walkley, J. W. (2003). Physical activity of adults with intellectual disability. Journal of Intellectual E Developmental Disability, 28, 323-334.

Thompson, J. R., Bryant, B. R., Campbell, E. M., Craig, E. M., Hughes, C. M., Rotholz, D. A., . . Wehmeyer, M. (2004). Supports Intensity Scale (SIS). Washington, DC: American Association on Mental Retardation.

Waninge, A., Evenhuis, I. J., van Wijck, R., \& van der Schans, C. P. (2011). Feasibility and reliability of two different walking tests in people with severe intellectual and sensory disabilities. Journal of Applied Research in Intellectual Disabilities, 24(6), 518-527. doi:10.1111/j.1468-3148.2011.00632.x

Zeno, S. A., Kim-Dorner, S., Deuster, P. A., Davis, J. L., Remaley, A. T., \& Poth, M. (2010). Cardiovascular fitness and risk factors of healthy African Americans and Caucasians. Journal of the National Medical Association, 102, 28-35.

C 2018 by the authors. Submitted for possible open access publication under the terms and conditions of the Creative Commons Attribution (CC BY) license (http://creativecommons.org/licenses/by/4.0/). 